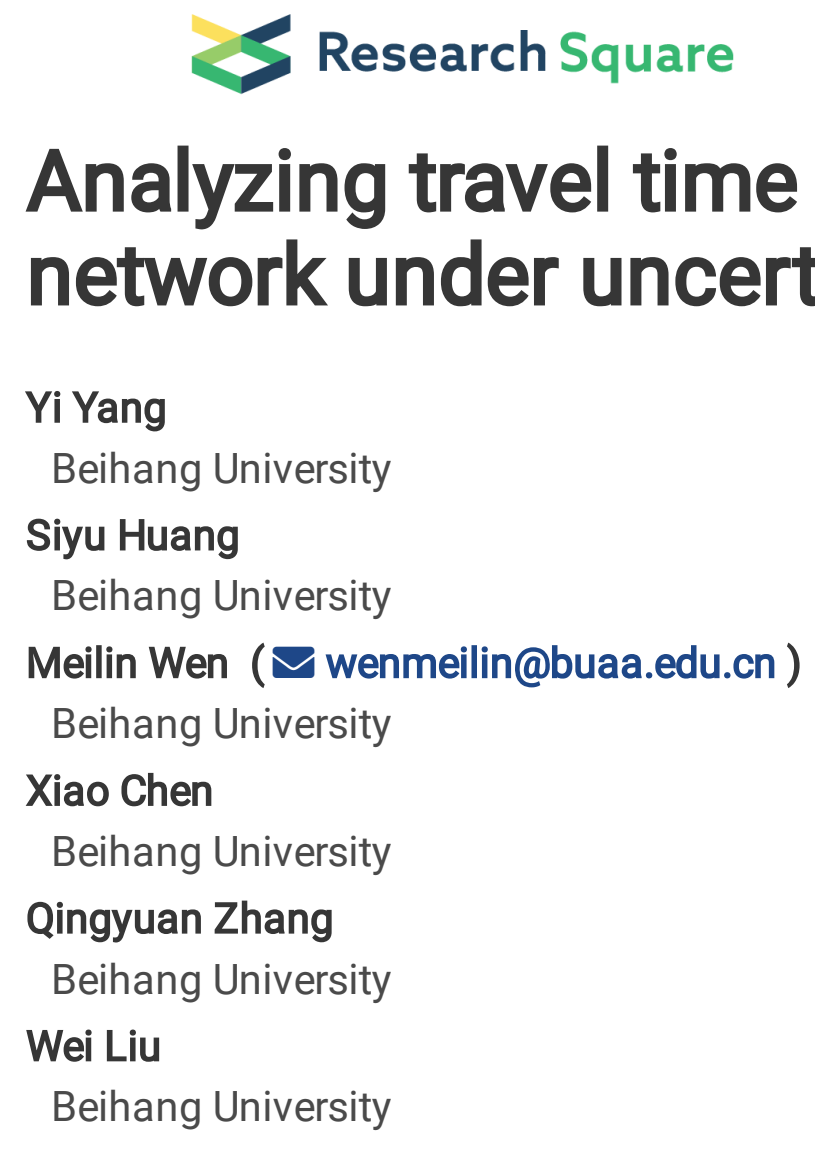

\author{
Yi Yang \\ Beihang University \\ Siyu Huang \\ Beihang University \\ Meilin Wen ( $\square$ wenmeilin@buaa.edu.cn ) \\ Beihang University \\ Xiao Chen \\ Beihang University \\ Qingyuan Zhang \\ Beihang University \\ Wei Liu \\ Beihang University
}

\title{
Analyzing travel time belief reliability in road network under uncertain random environment
}

\section{Research Article}

Keywords: Uncertainty Quantification, Travel Time Reliability, Belief Reliability, Uncertain Random System

Posted Date: April 6th, 2021

DOl: https://doi.org/10.21203/rs.3.rs-248701/v1

License: (c) (i) This work is licensed under a Creative Commons Attribution 4.0 International License.

Read Full License 


\title{
Analyzing travel time belief reliability in road network under uncertain random environment
}

YI YANG ${ }^{(1)}$, SIYU HUANG ${ }^{(1)}$, MEILIN WEN ${ }^{(1)}$, XIAO CHEN $^{(1)}$ QINGYUAN ZHANG ${ }^{2}$, WEI LIU ${ }^{1}$

(1) School of Reliability and Systems Engineering, Beihang University, Beijing 100191, China

(2) School of Aeronautic Science and Engineering, Beihang University, Beijing 100191, China

Corresponding author: Meilin Wen(wenmeilin@buaa.edu.cn)

\begin{abstract}
It is necessary to understand the operation status of the urban road network, especially when the network is complicated and uncertain. Taking travel time data as the starting point, we have studied the shortcomings of existing travel time reliability indicators. Most of them simplify or even ignore the information of traffic performance thresholds. According to the characteristics of the real urban road network, by extracting the information of the subject and object of the traffic service, we proposed measurement of the reliability of travel time in an uncertain random environment, that is, the travel time belief reliability, which takes the impact of the epistemic and random uncertainty on reliability into account. Next, we established the belief reliability model
\end{abstract}


of travel task under the uncertain random road environment. The model considers path selection, departure status and road conditions, and gives a path selection algorithm under time-varying road network. Besides, using the uncertainty regression analysis method, we explored the impact of road objective factors and driving state factors on the travel time threshold. Finally, we took the actual travel task in Beijing as an example to verify the feasibility and practicability of the model and algorithm.

Keywords: Uncertainty Quantification; Travel Time Reliability; Belief Reliability; Uncertain Random System.

\section{Introduction}

In recent years, urban road traffic has become more and more complex, and travelers not only have connectivity and short-term requirements for road traffic services, but also pay more and more attention to travel reliability. Sometimes, they even sacrifice travel time to ensure high travel reliability. Travel time reliability is an indicator that measures the stability of travel time from the perspective of time, reflecting the resilience of the road network under random and fluctuating traffic conditions. The reliability of travel time is very important to travelers, especially commercial transporters, because it can help them manage their time efficiently and even increase their income.

Many different quantifiable measures based on different definitions of travel time reliability have been proposed. They can be divided into two main categories according 
to the calculation method: Statistic measures and probability measures.

Statistics refers to the function of samples without unknown parameters. Statistical measurement is the general term for a series of indicators obtained by statistical analysis of travel time data. The reliability indicators of travel time proposed by many researchers are statistical measures. For example, Herman(Herman and Lam 1974), Sterman(Sterman and Schofer 1976), Bell(Bell and Iida 1997) and Bates(Bates et al. 2001) take the variability of travel time as a reliability index, that is, using divergence to measure the stability of travel time. Standard deviation(Hollander 2006; Hollander 2010), coefficient of variation (Sun et al. 2016) and the inverse of standard deviation(Sterman and Schofer 1976) are representative indexes. The buffer time is a waste of time caused by passengers who want to arrive on time and leave early. The buffer time is usually calculated by subtracting the average value from $95 \%$ or $90 \%$ of the travel time(Sun 2018; Xiong 2006; Zegeer et al. 2014). Tardy trips measure(Lomax and Margiotta 2003) represent the travel time unreliability by using the number of trips that results in late arrivals, such as the misery index (MI) (Lomax and Margiotta 2003), which measures the difference between the longest travel time and the mean travel time.

The statistical measurements are useful for managers because they have the characteristics of simple calculations, but they are not intuitive to travelers because they only focus on the macroscopic characteristics of travel time. For example, a standard deviation value of 0.5 may confuse the traveler and make little sense to him/her. When the buffer time is 30 minutes, the advice given to travelers is that it is more reliable to 
start 30 minutes earlier, but it does not directly reflect the reliability of the completion of the trip.

The probability measures are based on probability theory. They define travel time reliability as the probability that the trip can be completed within the acceptable time under a certain service level of road network service(Asakura and Kashiwadani 1991; Zhihua et al. 2004).

Compared with statistical measures, probabilistic measures retain the measure of travel time volatility, while also providing more intuitive reliability indexes. It is worth noting that the key to the use of such measures is to determine the acceptable time. In previous studies, it was regarded as a constant or multiple of standard deviation or quantile of travel time. However, this is actually a helpless method because it lacks information on the service level of the road network in the definition and also ignores information on the acceptability of travelers, which will impact on the acceptable time threshold(Xiaofei et al. 2014). In the previous literature, these travel time reliability measures are generally analyzed though travel time data. They extracted information about the performance of urban road traffic, but simplified or ignored the road facility factors and the traveler's travel status factors. However, one of them is the main body of the service provided by the urban road traffic system, and the other is the object which is to be served. Both of them are indispensable in the reliability measurement of the traffic network system.

We further analyze the service characteristics of the urban road traffic network. 
On the one hand, because of the random fluctuations in travel demand, there is an inherent randomness within the system, which is intuitively manifested in the fact that travel time will be different even under the same travel conditions. On the other hand, the main service target of the transportation network is travelers, and the travelers' cognition of the transportation network and travel status will affect the reliability measurement. For example, travelers do not have sufficient knowledge of road information that maybe this road is connected to a hospital. They may underestimate the time of the travel task; people with different travel purposes will have different evaluations of the reliability of the same road. The objective factors of the road and the subjective factors of travelers make the reliability measurement epistemically uncertain. Under the uncertain random environment, we need a new reliability measure that takes both epistemic uncertainty and random uncertainty into account.

For this reason, a mathematical theory called uncertainty theory is utilized to measure the travel time reliability. The uncertainty theory was founded in 2007 by Liu(Liu 2010) and subsequently refined by many pieces of research. The general purpose of the theory is to rationally deal with belief degrees evaluated by domain experts or related people. Belief degree is a way to describe an indefinite quantity, and its counterpoint is frequency generated by samples related to the probability theory. The uncertainty theory has been viewed as an appropriate mathematical system to model epistemic uncertainty and applied in various fields, including statistical analysis, risk analysis, differential equations, optimization problems, etc. In 2010, Liu first described 
reliability as an uncertain measure mathematically and provided some formulas about the reliability of Boolean systems. Later, Zeng(Zeng et al. 2013) named it the belief reliability and proposed an analysis method for unit level and product level. Then, the chance theory was proposed by Liu(Liu 2013b) in 2013. It is a combination of uncertainty theory and probability theory, which can be used to analyze systems with random uncertainty and cognitive uncertainty. In 2019, Kang(Kang 2020) finally proposed a theoretical framework for belief reliability.

The rest of this paper introduces the basic definitions and theorems of uncertainty theory and chance theory. In section 3 , we give the definition and calculation formula of travel time belief reliability. In section 4, we establish a travel time model for travel tasks in the urban road network. Then, the uncertainty regression model of the travel time threshold is established in section 5. Finally, we give an example to verify the travel time belief reliability measure.

\section{Preliminaries}

In this section, some basic definitions and theorems of uncertainty theory and chance theory will be introduced, which are the mathematical foundation of the travel time belief reliability. In addition, uncertain regression models and parameter solving methods are also introduced to provide instructions for the establishment of the following model. 


\subsection{Uncertainty theory and Chance theory}

The uncertainty measure represents the belief degree of the occurrence of an event, and the measure satisfies normality, duality, subadditivity axioms and product axioms. Definition 2.1 (Uncertain Variable) (Liu 2007) An uncertain variable is a function $\xi$ from an uncertainty space $(\Gamma, \mathcal{L}, \mathcal{M})$ to the set of real numbers such that $\{\xi \in B\}$ it is an event for any Borel set B of real numbers.

Definition 2.2 (Uncertainty distribution) (Liu 2007)The uncertainty distribution $\xi$ of an uncertain variable $»$ is defined by

$$
\Phi(x)=\mathcal{M}\{\xi \leq x\}
$$

for any real number $\mathrm{x}$.

In order to deal with the random uncertainty and epistemic uncertainty in complex systems, this paper will use the chance theory which was proposed by Liu ${ }^{(\operatorname{Liu} 2013 \mathrm{c})}$ in 2013, The chance measure is defined based on the chance space as follows:

Definition 2.3 (Chance Space) (Liu 2010) Let $(\Gamma, \mathcal{L}, \mathcal{M})$ be an uncertainty space and let $(\Omega, \mathcal{A}, \operatorname{Pr})$ be a probability space. Then the product $(\Gamma, \mathcal{L}, \mathcal{M}) \times(\Omega, \mathcal{A}, \operatorname{Pr})$ is called a chance space.

Definition 2.4 (Chance Measure) (Liu 2010) Let $(\Gamma, \mathcal{L}, \mathcal{M}) \times(\Omega, \mathcal{A}, \operatorname{Pr})$ be a chance space and let $\Theta \in L \times A$ be an event. Then the chance measure of $£$ is defined as

$$
C h(\Theta)=\int_{0}^{1} \operatorname{Pr}\{\omega \in \Omega \mid \mathcal{M}\{\gamma \in \Gamma \mid(\gamma, \omega) \in \Theta\} \geq x\} d x
$$

Definition 2.5 (Uncertain Random Variable) (Liu 2010)An uncertain random 
variable is a function $\xi$ from a chance space $(\Gamma, \mathrm{L}, \mathrm{M}) \times(\Omega, \mathrm{A}, \mathrm{Pr})$ to the set of real numbers such that is an event in $\mathrm{L} \times \mathrm{A}$ for any Borel set $\mathrm{B}$.

Definition 2.6 (Chance Distribution)(Liu 2010) Let $\xi$ be an uncertain random variable.

Then its chance distribution is defined by

$$
\Phi(x)=C h\{\xi \leq x\}
$$

for any $x \in R$

To obtain the chance distribution of the uncertain random variable, some operational laws will be used:

Theorem 2.1(Liu 2010)Let $\xi_{1}, \xi_{2}, \cdots, \xi_{n}$ be independent uncertain variables with regular uncertainty distributions $\Phi_{1}, \Phi_{2,}, \cdots, \Phi_{n}$, respectively. If the function $f\left(x_{1}, x_{2}, \cdots, x_{n}\right)$ is strictly increasing with respect to $x_{1}, x_{2}, \cdots, x_{m}$ and strictly decreasing with respect to $x_{m+1}, x_{m+2}, \cdots, x_{n}$, then the uncertain variable

$$
\xi=f\left(\xi_{1}, \xi_{2}, \cdots, \xi_{n}\right)
$$

has an inverse uncertainty distribution.

$$
\Psi^{-1}(\alpha)=f\left(\Phi_{1}^{-1}(\alpha), \cdots, \Phi_{m}^{-1}(\alpha), \Phi_{m+1}^{-1}(1-\alpha), \cdots \Phi_{n}^{-1}(1-\alpha)\right)
$$

Theorem 2.2(Liu 2013a) Let $\eta_{1}, \eta_{2}, \cdots, \eta_{m}$ are independent random variables with probability distributions $\Psi_{1}, \Psi_{2}, \cdots, \Psi_{m}$, respectively, and let $\tau_{1}, \tau_{2}, \cdots \tau_{n}$ be uncertain variables (not necessarily independent). Then the uncertain random variable

$$
\xi=f\left(\eta_{1}, \eta_{2}, \cdots, \eta_{m}, \tau_{1}, \tau_{2}, \cdots \tau_{n}\right)
$$

has a chance distribution

$$
\Phi(x)=\int_{R^{m}} M\left\{f\left(y_{1}, y_{2}, \cdots, y_{m}, \tau_{1}, \tau_{2}, \cdots \tau_{n}\right) \leq x\right\} d \Psi_{1}\left(y_{1}\right) \cdots d \Psi_{m}\left(y_{m}\right)
$$


for any number $\mathrm{x}$.

\subsection{Uncertain regression analysis}

Uncertain regression analysis is a statistical analysis method for uncertain variables, and it is mainly used to analyze the relationship between dependent variables and independent variables.

Let $\left(x_{1}, x_{2}, \cdots, x_{p}\right)$ be a vector of explanatory variables, and let $y$ be a response variable. Suppose the relationship between $\left(x_{1}, x_{2}, \cdots, x_{p}\right)$ and $y$ can be expressed by a function $\mathrm{f}$. That is, the regression model is given as

$$
y=f\left(x_{1}, x_{2}, \cdots, x_{p} \mid \boldsymbol{\beta}\right)+\varepsilon
$$

where $\boldsymbol{\beta}$ is a vector of unknown parameters and $\varepsilon$ is a disturbance term.

Theorem 2.3 (Lio and Liu 2020)Let $\left(x_{i 1}, x_{i 2}, \cdots, x_{i p}, y_{i}\right), i=1,2, \cdots n$ be a set of observed values, and let the regression model be

$$
y=f\left(x_{1}, x_{2}, \cdots, x_{p} \mid \boldsymbol{\beta}\right)+\varepsilon
$$

where $\varepsilon$ is a normal uncertain variable $\mathcal{N}(e, \sigma)$ with unknown parameters e and $\sigma$. Then, the maximum likelihood estimator $\left(\boldsymbol{\beta}^{*}, e^{*}, \sigma^{*}\right)$ solves the maximization problem,

$$
\max _{\beta, e, \sigma} L\left(\boldsymbol{\beta}, e, \sigma \mid z_{1}, z_{2}, \ldots, z_{n}\right)
$$

where 


$$
L\left(\boldsymbol{\beta}, e, \sigma \mid z_{1}, z_{2}, \ldots, z_{n}\right)=\frac{\frac{\pi}{\sqrt{3} \sigma} \exp \left(\frac{\pi}{\sqrt{3} \sigma} \vee^{i=1}\left|e-z_{i}\right|\right)}{\left(1+\exp \left(\frac{\pi}{\sqrt{3} \sigma^{i=1}}{ }^{n}\left|e-z_{i}\right|\right)\right)^{2}}
$$

and $z_{i}=y_{i}-f\left(x_{i 1}, x_{i 2}, \cdots, x_{i p} \mid \boldsymbol{\beta}\right), i=1,2, \cdots, n$.

Furthermore, $\left(\boldsymbol{\beta}^{*}, e^{*}\right)$ solves the minimization problem,

$$
\min _{\boldsymbol{\beta}, e} \underset{i=1}{\stackrel{n}{\vee}}\left|e-y_{i}+f\left(x_{i 1}, x_{i 2}, \cdots, x_{i p} \mid \boldsymbol{\beta}\right)\right|
$$

and $\sigma^{*}$ solves the maximization problem,

$$
\max _{\sigma>0} \frac{\frac{\pi}{\sqrt{3} \sigma} \exp \left(\frac{\pi}{\sqrt{3} \sigma} \mathrm{v}^{\mathrm{v}}\left|e^{*}-y_{i}+f\left(x_{i 1}, x_{i 2}, \ldots, x_{i p} \mid \beta^{*}\right)\right|\right)}{\left(1+\exp \left(\frac{\pi}{\sqrt{3} \sigma} \mathrm{v}^{n}\left|e^{*}-y_{i}+f\left(x_{i 1}, x_{i 2}, \ldots, x_{i p} \mid \beta^{*}\right)\right|\right)\right)^{2}}
$$

\section{Travel time belief reliability measure}

In this section, a new measure of travel time reliability under uncertain random environment called travel time belief reliability will be introduced. We will give calculation formulas and numerical solution algorithms for new indicators

\subsection{Performance analysis}

Travel time is one of the most common and intuitive indexes in the transportation system. At present, there are many advanced technologies and detection instruments in the traffic administrative department, so it is not difficult to obtain travel time data, and using big data mining methods to process these data is also a research hotspot. We assume that travel time is a random variable. With such a large sample size, it can be 
considered that the frequency histogram of travel time is very close to the real density curve, which is in line with the premise of applying probability theory. Travel time reliability is a measure that reflects the quality of the service.

For travelers, reliable service is that the travel time is within their acceptable range. It is worth noting that this range may not necessarily have a lower limit, because maybe for travelers, the shorter the travel time, the better. Even if there is a lower limit, people are more willing to accept the loss of travel time below the lower limit. In this paper, the upper limit of the acceptable time range is called the travel time threshold. The travel time threshold is a variable that is affected by many external factors, for example, for travel purposes, traveler's perception of the road, the choice of departure time, and the objective state of the road. This also makes the travel time threshold complicated, and our knowledge of its true value is insufficient. In addition, due to human participation, the information we collect about the threshold is subjective. These lead to epistemic uncertainty in the model. Therefore, we assume that the travel time threshold is an uncertain variable.

We define $t$ as the travel time and notate the accepted travel time threshold as $T$. The travel time is a Smaller-the-better (STB) parameters(Zhang et al. 2018), which means the trip will fail when travel time is higher than the threshold. Then, the travel time reliable criterion is as follows:

$$
t_{a}<T_{a}
$$

Namely, the trip is reliable for travelers when the criterion is met, and the failure 
criterion is the opposite, which is the same as the reliability of the engineering industry.

\subsection{Travel Time Belief Reliability (TTBR)}

According to the above performance analysis, the definition of travel time belief reliability as follows:

Definition 3.1 Travel Time Belief Reliability (TTBR): Travel time belief reliability is a chance measure that travelers can reach their destination within their acceptable time threshold, the mathematical expression of travel time belief reliability as follows:

$$
R_{B}=C h(t<T)
$$

Where $t$ is the travel time and obeys the probability distribution $F(t) . T$ is the travel time threshold and obeys the uncertainty distribution $\Phi(\tau)$.

Then, the travel time belief reliability can be calculated as the formula:

$$
\begin{aligned}
R_{B} & =C h\{t \leq T\} \\
& =C h\{t-T \leq 0\} \\
& =\Psi(0)
\end{aligned}
$$

According to the chance theory and the theorem 2.2 mentioned in part 2.1 and 2.2.

we have the chance distribution:

$$
\begin{aligned}
\Psi(x) & =C h(t-T \leq x) \\
& =\int M\{t-T \leq x\} d F(t)
\end{aligned}
$$

Where $t-T$ is the performance margin of travel time.

When analyzing the urban route network, a travel task may involve multiple road sections or multiple alternative routes. At this time, the theoretical calculation may be 
difficult. We also give a numerical calculation method based on the structure function as follows:

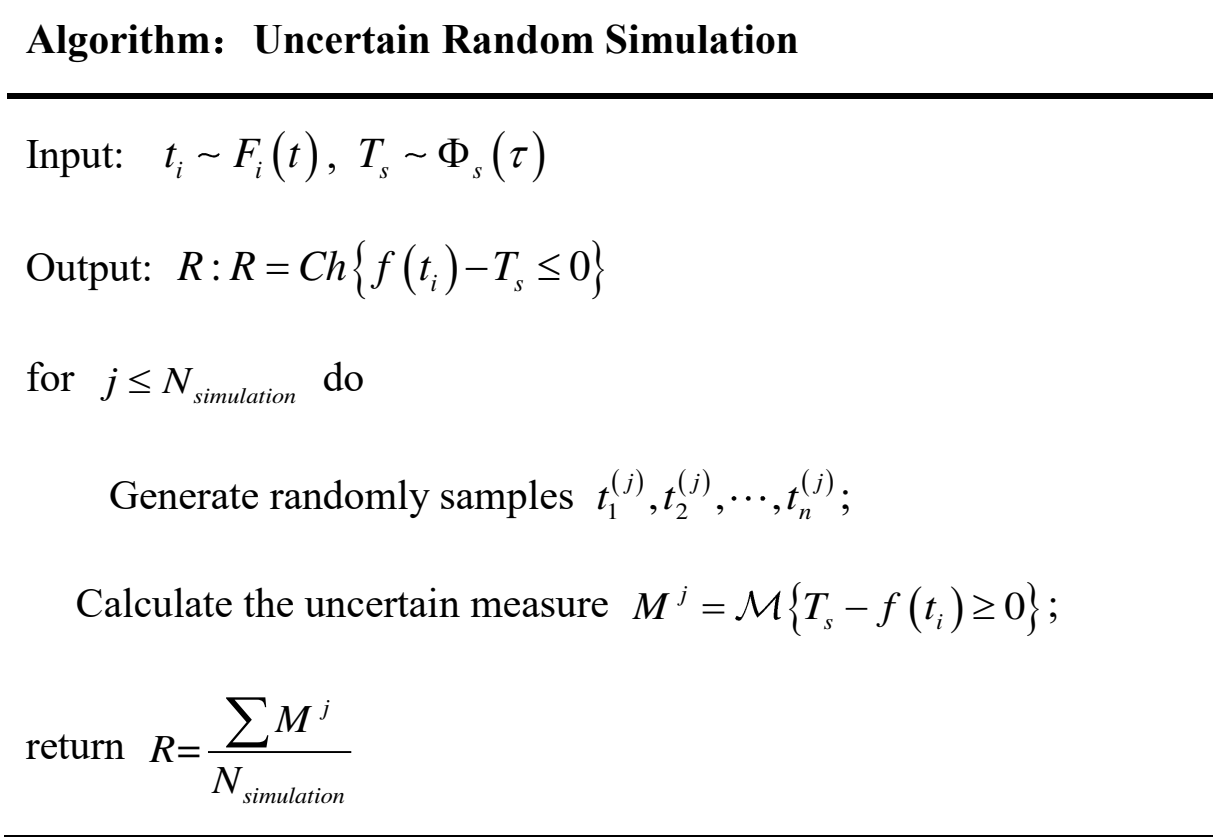

\section{Distribution of travel time in the urban road network}

In this section, we consider the real travel situation, and analyze the travel time of a certain travel task, that is from an origin to a destination. In the following, we will call it an OD pair. Then, we use a more sensitive goodness-of-fit test to determine the probability distribution function of the travel time.

\subsection{Improved K-Shortest-Path choice algorithm of the urban road network}

In practical travel, travelers have to face the traffic information of the whole region.

The route to the destination may be different because the travelers' choices are not fixed.

An important work is route choice. Once the path is determined, the network problem 
can be reduced to an OD pair problem by reducing the dimension. However, traffic network is a time-dependent network, that is to say, its state changes over time. We establish the $\mathrm{k}$ shortest path model for this time-dependent random network.

We assume a traffic network with roads as edges and intersections as nodes. Travelers have an exact starting point and destination, which can be represented by network nodes. Moreover, travelers are provided with at least $\mathrm{k}$ routes to their destination before departure.

Some symbols are expressed as follows:

$(V, E \times T, G):$ the traffic network;

$\mathrm{V}$ : the collection of $n$ nodes;

$E$ : the collection of $N$ links;

$T=\left[\tau_{0}, \tau_{n}\right]:$ the valid span on which we concerned;

$G=\left(g_{i j}\right):$ the matrix of the side weight function;

$g_{i j}(t)$ : travel time from node $\mathrm{i}$ to $\mathrm{j}$ at departure time $\mathrm{t}$;

$f_{i(t)}:$ the travel time from node $v_{i}$ to end node $v_{N}$ departed at $\mathrm{t}$;

$A_{(j)}^{-1}:$ a collection of start nodes $v_{i}$ of the link $\widehat{e}_{i j}$;

$\operatorname{Succ}(i)=j$ : the successor node $v_{i}$ is $v_{j}$.

Divide $T$ into $M$ parts by tiny interval time $\Delta$, let $S=\left\{\tau_{0}, \tau_{0}+\Delta, \tau_{0}+2 \Delta, \cdots, \tau_{0}+(M-1) \Delta\right\}$. When $\Delta$ is small enough, the expected value of each interval can be used to replace the travel time, which can also reflect the timedependent of the traffic network. 
A k-shortest paths (KSP) algorithm based on improved Dijkstra(Fen et al. 2008) is proposed, which can get the k-shortest travel time paths in the sense of expected value.

\section{Algorithm: Improved KSP Algorithm}

\section{PartI: Dijkstra algorithm of time-dependent stochastic networks}

Input: $\quad f_{i(t)}=\left\{\begin{array}{ll}\infty & i \neq N \\ 0 & i=N\end{array}\right.$, List $=\left\{v_{N}\right\}$

Output: Succ

While List $\neq \varnothing$ do

$$
\begin{aligned}
& \text { Choose } v_{j}=\operatorname{List}(1) \\
& \text { For } \forall v_{i} \in A_{(j)}^{-1} / v_{j} \text { do } \\
& \text { For } \forall t \in S=\left\{\tau_{0}, \tau_{0}+\Delta, \tau_{0}+2 \Delta, \cdots, \tau_{0}+(M-1) \Delta\right\} \text { do } \\
& \text { If } f_{i}(t)>g_{i j}(t)+f_{i}\left(t+g_{i j}(t)\right) \text { do } \\
& \qquad f_{i}(t)=g_{i j}(t)+f_{i}\left(t+g_{i j}(t)\right) \\
& \operatorname{succ}(i)=j \\
& \text { List }=\left\{\text { List }, v_{i}\right\}
\end{aligned}
$$

return Succ

\section{PartII: Find K-shortest paths}

Input: $\operatorname{Graph}(V, E \times T, G)$, origin node and destination node

Output: K-shortest travel time paths

Get the shortest path $P_{k}(k=1)$ based on improved Dijkstra algorithm

For $k=1,2, \cdots K$ do 
For $\forall v_{i} \in P_{k} / v_{n}$

Let $g_{i, i+1}=\infty$

Get the shortest path $P_{i}^{\prime}$ from $v_{i}$ to $v_{n}$

$$
P_{k}=\min \left\{P_{i}^{\prime}\right\}
$$

return $P_{k}(k=1,2, \cdots K)$

Through the above algorithm, we can get K shortest travel time paths in the sense of expected value. We assume that travelers are sensible decision-makers, and the goal is to keep the trip as short as possible. Systematically, the $\mathrm{K}$ paths are in parallel, and the margin of stroke time is the difference between the minimum stroke time and the threshold. Therefore, the travel time of the travel network is

$$
t_{s}(\tau)=\min \left[t_{i}(\tau)\right](i=1,2 \cdots, k)
$$

Where $i(i=1,2 \cdots, k)$ means the traveler chooses the $i^{\text {th }}$ path, $\tau$ is the departure time.

\subsection{Cumulative effect of travel time}

In real situations, the entire transportation network is a network that changes dynamically over time. Travel is a time-dependent behavior. Every time a traveler finishes a link of the path, and reach the next link, the time will be cumulated, and the state of the two links will change.

We assume that the $i^{\text {th }}$ path is composed of some independent links. The travel time of each link is known, but it cannot be added directly. Assuming that the traveler's 
departure time is $\tau_{0}$. Then the travel time of the $i^{\text {th }}$ path can be calculated by

$$
t_{i}\left(\tau_{0}\right)=t_{i 1}\left(\tau_{0}\right)+t_{i 2}\left(E\left(t_{i 1}\right)+\tau_{0}\right)+\cdots+t_{i n_{i}}\left(\sum_{j=1}^{n_{i}-1} E\left(t_{i j}\right)+\tau_{0}\right)
$$

Where $t_{i j}\left(i=1,2, \cdots, k ; j=1,2 \cdots n_{i}\right)$ is the travel time of link $\mathrm{j}$ in $i^{t h}$ path, which obeys the random distributions $F_{i j}(t), t_{i j}(\tau)$ represents the travel time though link $\mathrm{j}$ at time $\tau$.

\subsection{Goodness-of-fit test of travel time distribution}

To determine the travel time probability distribution, this paper uses the nonparameter test method to find the probability distribution function of travel time. First, we divided the 24 hours of a day into n parts by $1440 / \mathrm{n}$ minutes and collected the travel time of vehicles leaving within $1440 / i(i=1,2, \cdots n)$ minutes. The observed samples $t_{i 1}, t_{i 2}, \cdots, t_{i N}$ are travel time of a road in the same i_th minutes of $\mathrm{N}$ days. Through the statistical analysis of these samples, we can get the daily travel time distribution pattern at the time 1440/i.

There are various distributions to model travel time in different situations(Aron et al. 2014). The lognormal distribution, the Gamma one, the Weibull one and the burr one are used(Al-Deek and Emam 2006; Lu and Dong 2018; Pu 2011; Taylor 2012). We use the za.test function in the "DistributionTest" package in $\mathrm{R}$ to test travel time probability distribution function, which is more sensitive and accurate to the drift of normal distribution and logarithmic normal distribution(Zhang 2002). If the test Pvalue passes the test, we will get the travel time distribution of one road. 
Finally, in the dynamic urban road network, the travel time distribution of a particular travel task is as follows:

$$
F_{s}(t)=1-\prod_{i=1}^{K}\left(1-F_{i}(t)\right)
$$

And according to the convolution formula, the $F_{i}(t)$ is as follows:

$$
F_{i}(t)=F_{i 1}(t) * F_{i 2}(t) * \ldots F_{i j}(t) * \ldots * F_{i n_{i}}(t)
$$

\section{Uncertain regression model of travel time threshold}

This section attempts to establish an uncertainty regression model based on travel time thresholds for different departure times.

In this uncertain regression model, the independent variable selects the objective state and travel state of the road, and the dependent variable is the travel time threshold evaluation given by the traveler. After a series of inquiries about historical travelers' tolerance for travel time on this road, many empirical data have been obtained. Nevertheless, these people cannot give a very accurate description, and even different people may have different reliability. What they say contains real information about the actual threshold.

We choose the eight factors of road grade: traffic speed, length, bus stops, traffic lights, intersections, commercial buildings nearby, and whether to travel during peak hours. Then, we collect some sample data through research. It is worth noting that the traffic speed data we collect may be inaccurate, but this will not lead to mathematical modeling problems because uncertain regression can be analyzed based on inaccurate 
data(Yao and Liu 2018).

Some symbols will be used in the model, and they are described below:

$x_{1}$ : road grade, which is determined by the road design department and based on the administrative distinction, See Table 1 for specific grades;

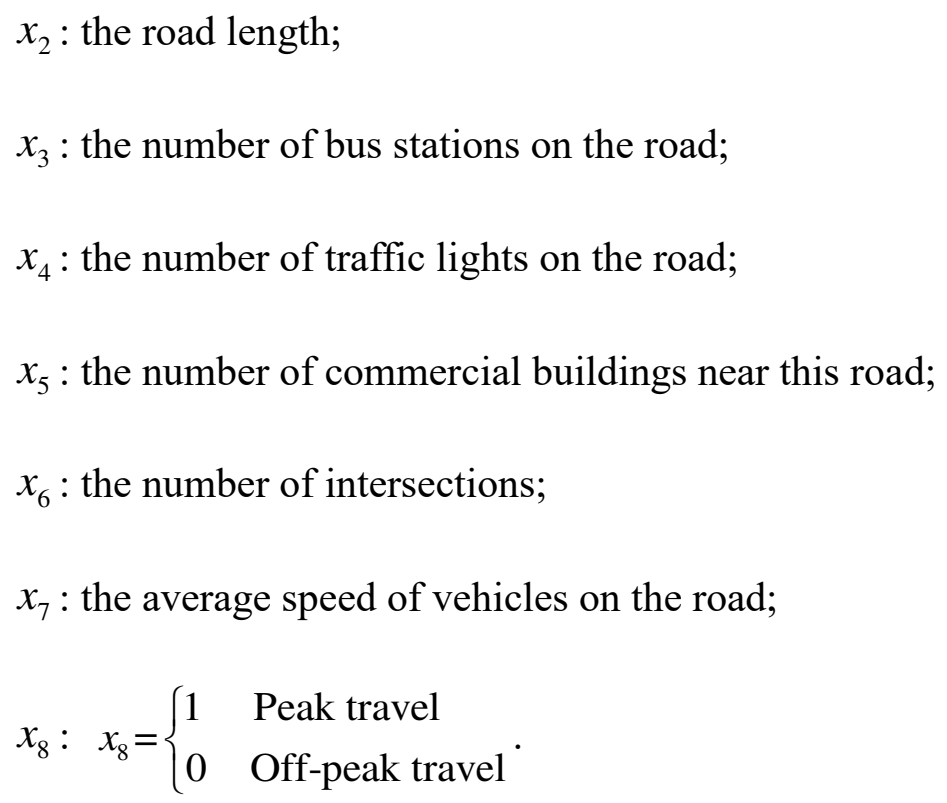

Assuming that the travel time threshold $\mathrm{T}$ follows a normal uncertainty distribution. The sensitivity of travelers to the departure time, and the peak and off-peak trips are considered separately.

An uncertain linear regression model with 7 factors and the travel time threshold is established:

$$
T=\beta_{0}+\sum_{j=1}^{7} \beta_{j} x_{j}+\varepsilon
$$

Where $\varepsilon$ obeys the normal uncertain variable $\mathcal{N}(0, \sigma), \hat{\boldsymbol{\beta}}$ solves the minimization problem, 


$$
\min _{\boldsymbol{\beta}, e} \underset{i=1}{\stackrel{n}{\vee}}\left|T_{i}-\beta_{0}-\sum_{j=1}^{7} \beta_{j} x_{j}\right|
$$

$\hat{e}=0$ and $\hat{\sigma}$ solves the maximization problem,

$$
\max _{\sigma>0} \frac{\frac{\pi}{\sqrt{3} \sigma} \exp \left(\frac{\pi}{\sqrt{3} \sigma}{ }^{i=1}{ }^{n}\left|T_{i}-\beta_{0}-\sum_{j=1}^{7} \beta_{j} x_{j}\right|\right)}{\left(1+\exp \left(\frac{\pi}{\sqrt{3} \sigma}{ }^{i}{ }^{n}\left|T_{i}-\beta_{0}-\sum_{j=1}^{7} \beta_{j} x_{j}\right|\right)\right)^{2}} .
$$

According to Theorem 2.3, the maximum likelihood estimation of the parameters can be obtained, and the uncertainty regression equation of the travel time threshold and its uncertainty distribution can be obtained:

$$
T=\hat{\beta}_{0}+\sum_{\mathrm{i}=1}^{7} \hat{\beta}_{j} x_{j}+\varepsilon
$$

where $\varepsilon \sim \mathcal{N}(0, \hat{\sigma})$ and $\hat{\sigma}$ satisfies formula (17).

Finally, we perform residual analysis to obtain the uncertainty distribution function of the travel time threshold:

$$
T \sim\left\{\begin{array}{lr}
\mathcal{N}_{1}\left(\hat{\beta}_{0}+\sum_{\mathrm{i}=1}^{7} \hat{\beta}_{j} x_{j}, \hat{\sigma}\right) & x_{8}=1 \\
\mathcal{N}_{0}\left(\hat{\beta}_{0}^{\prime}+\sum_{\mathrm{i}=1}^{7} \hat{\beta}_{j}^{\prime} x_{j}, \hat{\sigma}^{\prime}\right) & x_{8}=0
\end{array}\right.
$$

Table 1: Administrative division of road grades

\begin{tabular}{|c|c|}
\hline Road Grade & Road Level \\
\hline 1 & Intercity Highway \\
\hline 2 & Urban Expressway \\
\hline 3 & National Highway \\
\hline
\end{tabular}




\begin{tabular}{|c|c|}
\hline 4 & Provincial Road \\
\hline 5 & County Road \\
\hline 6 & Township Road / Other \\
\hline
\end{tabular}

\section{Case study}

In this section, we try to analyze the reliability of a specific travel task from an origin to a destination. The actual map of the area we studied is shown in Figure 1, and the blue dots are the traffic detectors. Furthermore, we collected travel speed data from July 27 to July 31 through these detectors.

First of all, we established the traffic network through the longitude and latitude data of the detectors and road geographic information. The simplified network is shown in Figure 2. The object of study is to acquire the travel time belief reliability from the origin $(116.3328,39.9929)$ to the destination $(116.3533,39.9765)$ in a working day. The distance between the two locations is about 4 kilometers. 
We collected the speed data between many monitoring points and preprocessed these data statistically to calculate the road travel time data. Furthermore, we studied the travel time of 288 time periods by dividing 24 hours at five-minute intervals. Then we use the za.test function in the "DistributionTest" package in R to test travel time probability distribution function. Finally, the travel time distribution function of each route and each time segment were obtained.

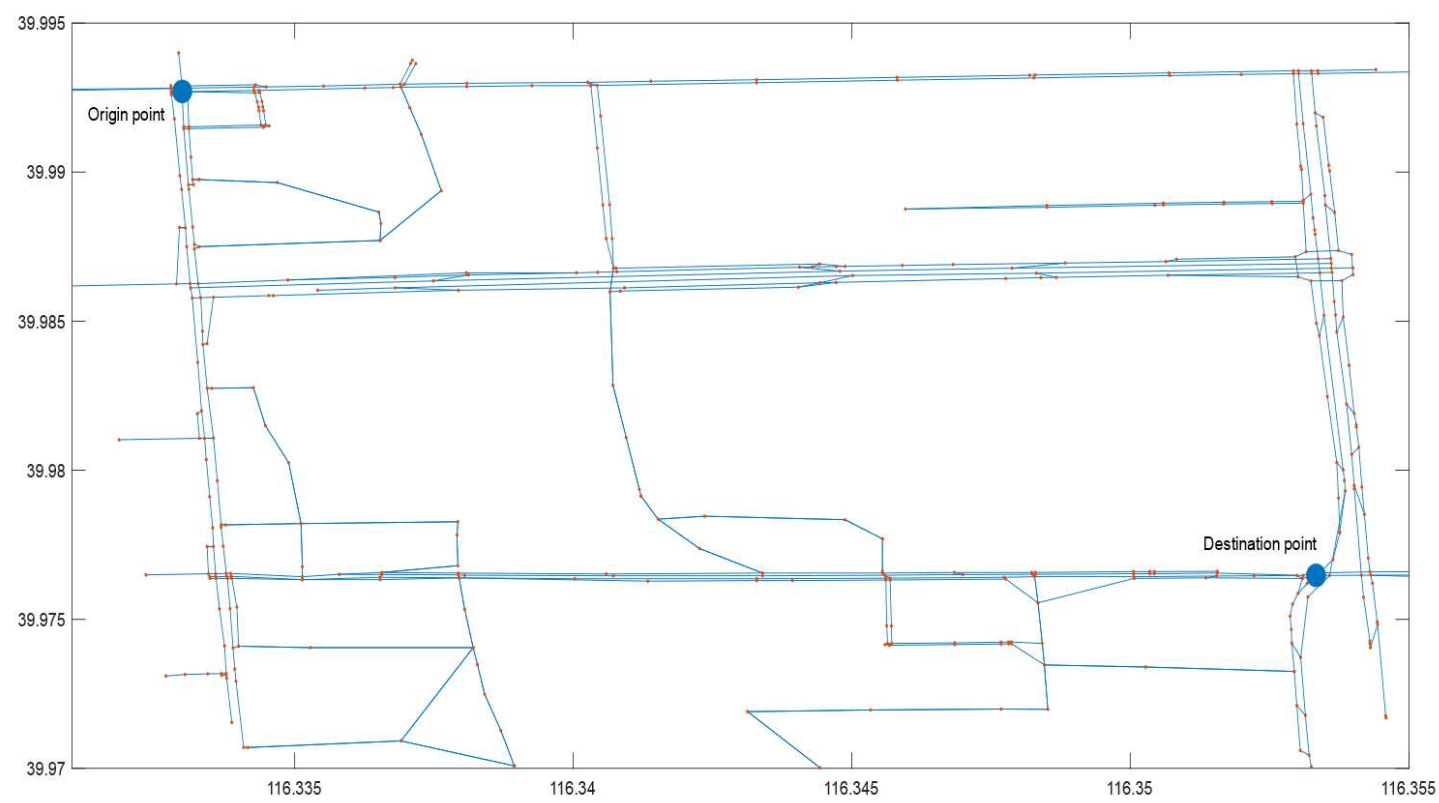

Figure1: The structure of the road network in the study area 


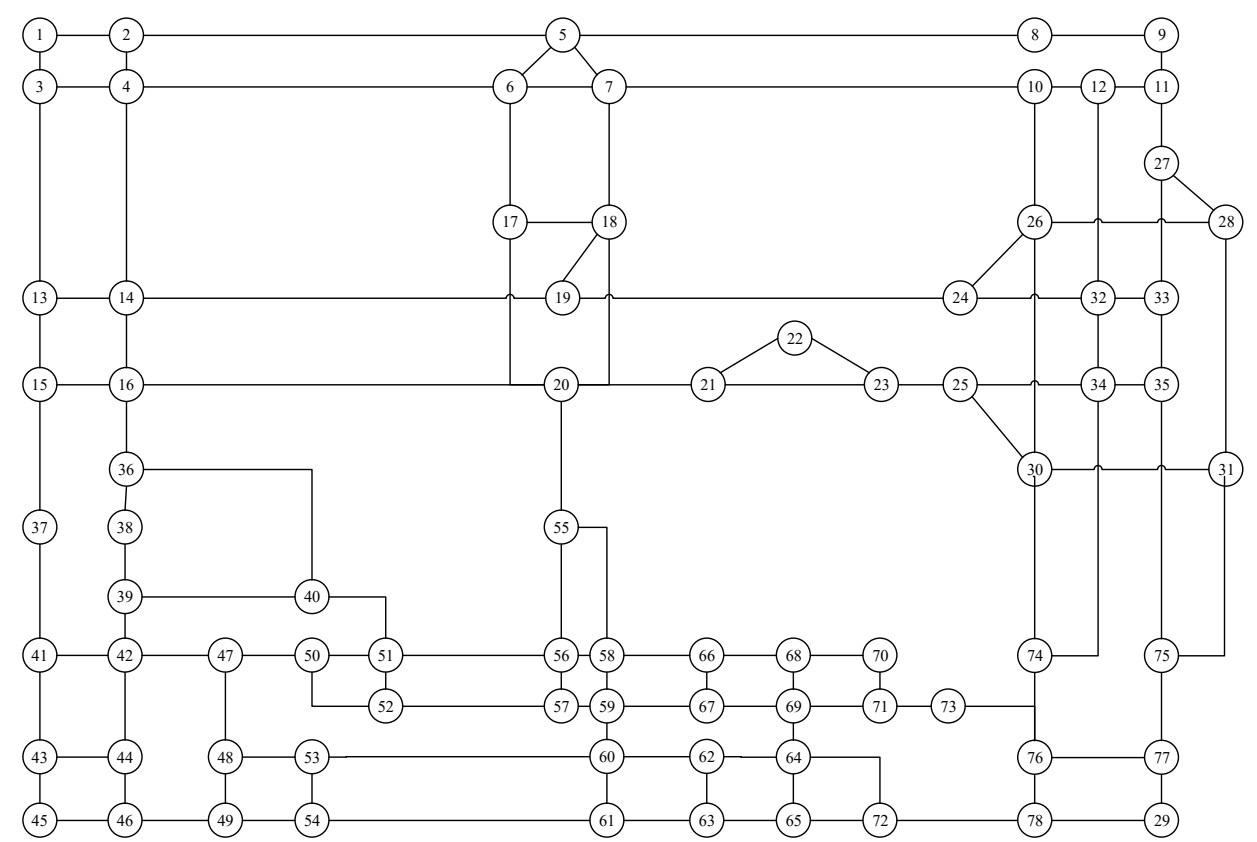

Figure2: The network topology of the study area

Next, we analyzed the travel time of all roads in the road network and used algorithm 1 to determine the real-time optimal route choices from the origin point to the destination in the sense of expected travel time. The travel demands of travelers in a whole day will change with time, which will lead to the change of the traffic capacity of the road network. In actual travel, the travelers will also choose different routes according to the departure time.

Table 2: The result of the K-shortest paths Algorithm

\begin{tabular}{ccccccccccccccccccccc}
\hline Paths & 110 \\
\hline 1 & 1 & 3 & 13 & 15 & 16 & 21 & 22 & 23 & 25 & 34 & 74 & 76 & 78 & 0 & 0 & 0 & 0 \\
2 & 1 & 3 & 13 & 15 & 16 & 21 & 23 & 25 & 34 & 74 & 76 & 78 & 0 & 0 & 0 & 0 & 0 \\
3 & 1 & 3 & 13 & 15 & 16 & 21 & 22 & 23 & 25 & 30 & 74 & 76 & 78 & 0 & 0 & 0 & 0 \\
\hline
\end{tabular}




\begin{tabular}{lllllllllllllllllll}
\hline 4 & 1 & 3 & 13 & 15 & 16 & 21 & 23 & 25 & 30 & 74 & 76 & 78 & 0 & 0 & 0 & 0 & 0 \\
5 & 1 & 3 & 4 & 6 & 17 & 20 & 55 & 56 & 57 & 59 & 60 & 62 & 64 & 72 & 78 & 0 & 0 \\
6 & 1 & 3 & 4 & 6 & 17 & 20 & 21 & 23 & 25 & 30 & 74 & 76 & 78 & 0 & 0 & 0 & 0 \\
7 & 1 & 3 & 4 & 6 & 17 & 20 & 21 & 22 & 23 & 25 & 34 & 74 & 76 & 78 & 0 & 0 & 0 \\
8 & 1 & 3 & 4 & 6 & 7 & 10 & 12 & 32 & 34 & 74 & 76 & 78 & 0 & 0 & 0 & 0 & 0 \\
9 & 1 & 3 & 4 & 6 & 7 & 10 & 26 & 30 & 74 & 76 & 78 & 0 & 0 & 0 & 0 & 0 & 0 \\
10 & 1 & 3 & 4 & 6 & 17 & 20 & 55 & 58 & 56 & 57 & 59 & 60 & 62 & 64 & 72 & 78 & 0 \\
11 & 1 & 3 & 13 & 15 & 37 & 41 & 43 & 45 & 46 & 49 & 54 & 53 & 60 & 62 & 64 & 72 & 78 \\
\hline
\end{tabular}

In this real research area, we can combine maps and field trips to collect information about road conditions. We collect data on the 11 shortest paths and important roads of the region in different periods. The main factors include road grade, road length, number of bus stops on the road, the number of signal lights, number of surrounding entertainment buildings, number of intersections, and average traffic speed. In particular, a path may consist of roads of multiple grades, and here we use the average grade of the road as the level of the entire path. For the travel time threshold, we surveyed 20 people who had travel experience in this area through a questionnaire and collected their acceptable time data for this travel at different departure times. Our observations are inaccurate, and the above indicators are all uncertain variables. Based on the data above, for the study area, we established an uncertain regression model of travel time thresholds for different departure times. 
Table 3: 11-shortest paths and operating status data of possible routes

\begin{tabular}{|c|c|c|c|c|c|c|c|c|}
\hline Path & Ave Grade & $\begin{array}{l}\text { Length } \\
\text { (m) }\end{array}$ & Bus & Light & Entertainment & Intersection & $\begin{array}{c}\text { Ave Speed } \\
\text { (Peak) }\end{array}$ & $\begin{array}{l}\text { Ave Speed } \\
\text { (Off-Peak) }\end{array}$ \\
\hline 1 & 3.67 & 3656.41 & 5 & 12 & 0 & 31 & 37.12 & 40.72 \\
\hline 2 & 4.00 & 3647.61 & 5 & 12 & 0 & 31 & 37.95 & 40.65 \\
\hline 3 & 3.75 & 3619.45 & 3 & 10 & 0 & 27 & 36.02 & 38.84 \\
\hline 4 & 4.09 & 3610.65 & 3 & 10 & 0 & 27 & 36.75 & 38.60 \\
\hline 5 & 5.64 & 3408.84 & 2 & 5 & 3 & 19 & 32.73 & 31.52 \\
\hline 6 & 4.42 & 3617.05 & 2 & 8 & 1 & 17 & 36.95 & 38.38 \\
\hline 7 & 4.00 & 3662.80 & 4 & 10 & 1 & 21 & 37.27 & 40.35 \\
\hline 8 & 4.27 & 3620.46 & 7 & 9 & 2 & 22 & 35.30 & 37.22 \\
\hline 9 & 4.50 & 3626.80 & 4 & 10 & 2 & 18 & 34.21 & 36.16 \\
\hline 10 & 5.67 & 3850.30 & 2 & 5 & 2 & 20 & 32.43 & 31.21 \\
\hline 11 & 5.69 & 3522.99 & 3 & 12 & 2 & 30 & 31.84 & 30.90 \\
\hline
\end{tabular}

Based on the state data of important roads in the area, we fitted the uncertainty regression equation of the travel time threshold near Beihang University in Haidian District.

$T=\left\{\begin{array}{lc}0.0170 x_{1}+0.0018 x_{2}-0.2111 x_{3}+0.0016 x_{4}+0.0018 x_{5}+0.0128 x_{6}+0.017 x_{7}-0.361 & \text { peak } \\ -0.0161 x_{1}+0.0019 x_{2}+0.0565 x_{3}-0.0081 x_{4}+0.0550 x_{5}-0.0198 x_{6}+0.0059 x_{7}-0.0161 & \text { off }- \text { peak }\end{array}\right.$

The residual analysis of the above two uncertain regression models can obtain the uncertainty distribution of the residual term: 


$$
\begin{aligned}
& \varepsilon_{1} \sim \mathcal{N}(0,1.5) \\
& \varepsilon_{0} \sim \mathcal{N}(0,0.65)
\end{aligned}
$$

Then the forecast uncertain variable of $\mathrm{T}$ concerning $\tilde{x}_{1}, \tilde{x}_{2}, \cdots, \tilde{x}_{7}$ is determined by

$$
T= \begin{cases}\hat{\beta}_{0}+\sum_{j=1}^{7} \hat{\beta}_{j} x_{j}+\varepsilon_{1} & x_{8}=1 \\ \hat{\beta}_{0}^{\prime}+\sum_{j=1}^{7} \hat{\beta}_{j}^{\prime} x_{j}+\varepsilon_{0} & x_{8}=0\end{cases}
$$

The travel time threshold $\mathrm{T}$ will be estimated by the above, and it is natural to define the uncertain expect:

$$
\begin{aligned}
& \mu_{1}=\hat{\beta}_{0}+\sum_{\mathrm{i}=1}^{7} \hat{\beta}_{j} E\left[\tilde{x}_{j}\right] \\
& \mu_{0}=\hat{\beta}_{0}^{\prime}+\sum_{\mathrm{i}=1}^{7} \hat{\beta}_{j}^{\prime} E\left[\tilde{x}_{j}\right]
\end{aligned}
$$

The uncertainty distribution of the travel time thresholds for these two different departure times is obtained:

$$
T \sim\left\{\begin{array}{cc}
\mathcal{N}(6.915,1.25) & \text { peak } \\
\mathcal{N}(7.143,0.63) & \text { off - peak }
\end{array}\right.
$$

We use hypothesis testing and uncertainty regression analysis to obtain the random distribution of travel time and the uncertainty distribution of travel time threshold.

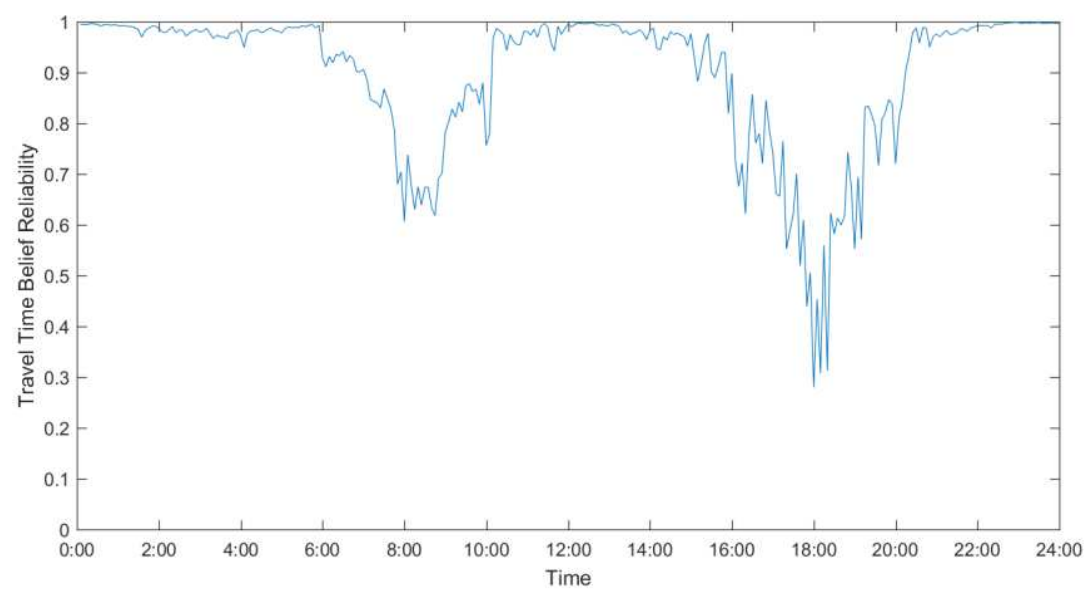


Furthermore, according to formulas 32 and 33, we can obtain an analytical solution for travel time reliability. Since calculating the travel time probability distribution in this example involves multiple convolutions, the calculation is more complicated, so we use an uncertain stochastic simulation algorithm to obtain a numerical solution. The simulation scale is 10,000 . Finally, the change of the OD's travel time belief reliability with depart time is shown in the Figure 3.

Figure 3: The variation of OD pair travel time belief reliability on working days

\section{Conclusion}

This study analyzed the existing travel time reliability of urban traffic and proposed a new measurement-- travel time belief reliability. In the previous literature, the travel time threshold is either a fixed window width or defined as the quantile of the probability travel time reliability index. We take epistemic factors such as the uncertainty of the traveler's knowledge of road condition information into account. It is assumed that the travel time threshold is a variable. We have also established an uncertain regression model of the travel time threshold, which can be used to analyze the impact of people's perception of objective road factors and operating conditions on the evaluation of travel time threshold. The obtained regression equation can be regarded as the empirical equation of the travel time threshold near the study area during working days. Based on the definition of reliability under the definition of performance margin, we give a new definition of the reliability of travel time and give 
the calculation formulas for the unit level and task level. Especially for more complex travel task structures, we also give numerical calculation methods for uncertain stochastic simulation.

The method based on uncertainty theory and chance theory has more basis in the establishment and analysis of reliability model It considers the impact of travelers' understanding of the road and the objective state of the road on reliability, and tries to solve the regret of insufficient information extracted by the existing reliability indicators by analyzing the uncertain random environment. On the one hand, the travel time reliability measurement based on belief data can reflect the wellbeing degrees of travelers. On the other hand, it provides pedestrians and urban traffic management departments with an intuitive index that is more in line with the real traffic environment, which is useful for travel planning and urban road congestion management.

Future research work can further analyze the objective factors of the road, extract more relevant features, and optimize the empirical function of the travel time threshold. In addition, we can also evaluate the overall belief reliability of the urban road network, and provide parameter support for solving the bottleneck problem of traffic congestion.

\section{References}

Al-Deek H, Emam EB (2006) New methodology for estimating reliability in transportation networks with degraded link capacities Journal of intelligent transportation systems 10:117-129

Aron M, Bhouri N, Guessous Y (2014) Estimating travel time distribution for reliability 
analysis Transportation Research Arena, TRA, paper 19638

Asakura Y, Kashiwadani M Road network reliability caused by daily fluctuation of traffic flow. In: PTRC Summer Annual Meeting, 19th, 1991, University of Sussex, United Kingdom, 1991.

Bates J, Polak J, Jones P, Cook A (2001) The valuation of reliability for personal travel Transportation Research Part E: Logistics and Transportation Review 37:191-229 doi:10.1016/s1366-5545(00)00011-9

Bell MG, Iida Y (1997) Transportation network analysis. John Willy and Sons. doi:10.1002/9781118903032.ch2

Fen L, Ying Z, Jie X, Zhaoxiang L (2008) Research of Dynamic Shortest Path on a Random Network Journal of the CUM(National Sciences Edition) 017:47-53

Herman R, Lam T (1974) Trip time characteristics of journeys to and from work Transportation traffic theory 6:57-86

Hollander Y (2006) Direct versus indirect models for the effects of unreliability Transportation Research Part A: Policy Practice 40:699-711

Hollander Y What Do We Really Know About Travellers' Response to Unreliability? In: Choice Modelling: The State-of-the-art and the State-of-practice-Proceedings from the Inaugural International Choice Modelling Conference, 2010. Emerald Group Publishing, p 461

Kang R (2020) Belief Reliability Theory and Methodology. National Defence Industry Press, Beijing

Lio W, Liu B (2020) Uncertain maximum likelihood estimation with application to uncertain regression analysis Soft Computing 24:9351-9360

Liu B (2007) Uncertainty theory. Uncertainty theory. Springer,

Liu B (2010) Uncertainty Theory. Studies in Computational Intelligence. Springer. doi:10.1007/978-3-642-13959-8

Liu Y (2013a) Uncertain random programming with applications Fuzzy Optimization Decision Making 12:153-169 
Liu Y (2013b) Uncertain random variables: a mixture of uncertainty and randomness Soft Computing 17:625-634

Liu Y (2013c) Uncertain random variables: a mixture of uncertainty and randomness Soft Computing 17:625-634

Lomax T, Margiotta R (2003) Selecting travel reliability measures. Citeseer,

Lu C, Dong J (2018) Estimating freeway travel time and its reliability using radar sensor data Transportmetrica B: Transport Dynamics 6:97-114

$\mathrm{Pu}$ W (2011) Analytic relationships between travel time reliability measures Transportation Research Record 2254:122-130 doi:10.3141/2F2254-13

Sterman BP, Schofer JL (1976) Factors affecting reliability of urban bus services Journal of Transportation Engineering 102

Sun S (2018) The study of the travel time reliability. Master, People's Public Security Unversity of China

Sun Y, Shi J, Schonfeld PM (2016) Identifying passenger flow characteristics and evaluating travel time reliability by visualizing AFC data: a case study of Shanghai Metro Public Transport 8:341-363

Taylor MA (2012) Modelling travel time reliability with the Burr distribution ProcediaSocial Behavioral Sciences 54:75-83

Xiaofei S, Xumei C, wenfeng L, Ying W, lin W (2014) Development of an evaluation system of the reliability of travel time of freeways and the thresholds of corresponding indicators Journal of Transport Information and Safety 32:58-63

Xiong Z (2006) Study on Theory and Method of travel Time Reliability about Road Network. PhD, School of Traffic and Transportation

Yao K, Liu B (2018) Uncertain regression analysis: an approach for imprecise observations Soft computing 22:5579-5582

Zegeer J et al. (2014) Incorporating travel time reliability into the Highway Capacity Manual. National Research Council (US). Transportation Research Board, Zeng Z, Wen M, Kang R (2013) Belief reliability: a new metrics for products' reliability 
Fuzzy Optimization Decision Making 12:15-27

Zhang J (2002) Powerful goodness-of-fit tests based on the likelihood ratio Journal of the Royal Statistical Society: Series B 64:281-294

Zhang Q, Kang R, Wen M (2018) Belief reliability for uncertain random systems IEEE Transactions on Fuzzy Systems 26:3605-3614

Zhihua X, Zhisheng Y, Chunfu S (2004) Travel Time Reliability in Road Network Associated with Road Section China safety Science Journal:84-87+81

\section{Declarations}

Funding

This work was supported by the National Natural Science Foundation of China [61871013] and [71671009].

Availability of data and material

Not applicable

Code availability

Not applicable

Conflict of interest:

The authors declare that there is no conflict of interest regarding the publication of this paper.

Ethical approval:

This article does not contain any studies with human participants or animals performed by any of the authors. 
Figures

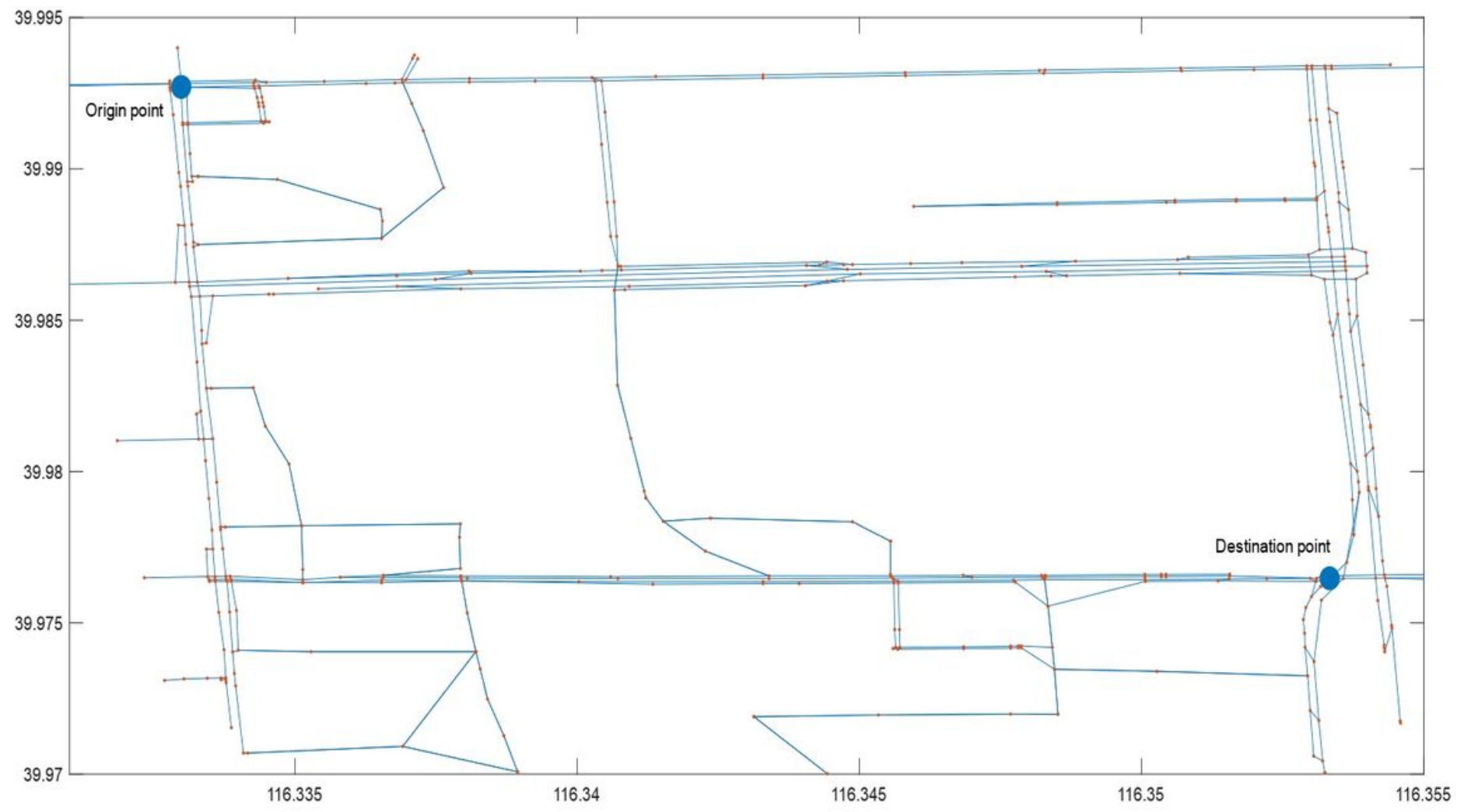

Figure 1

The structure of the road network in the study area 


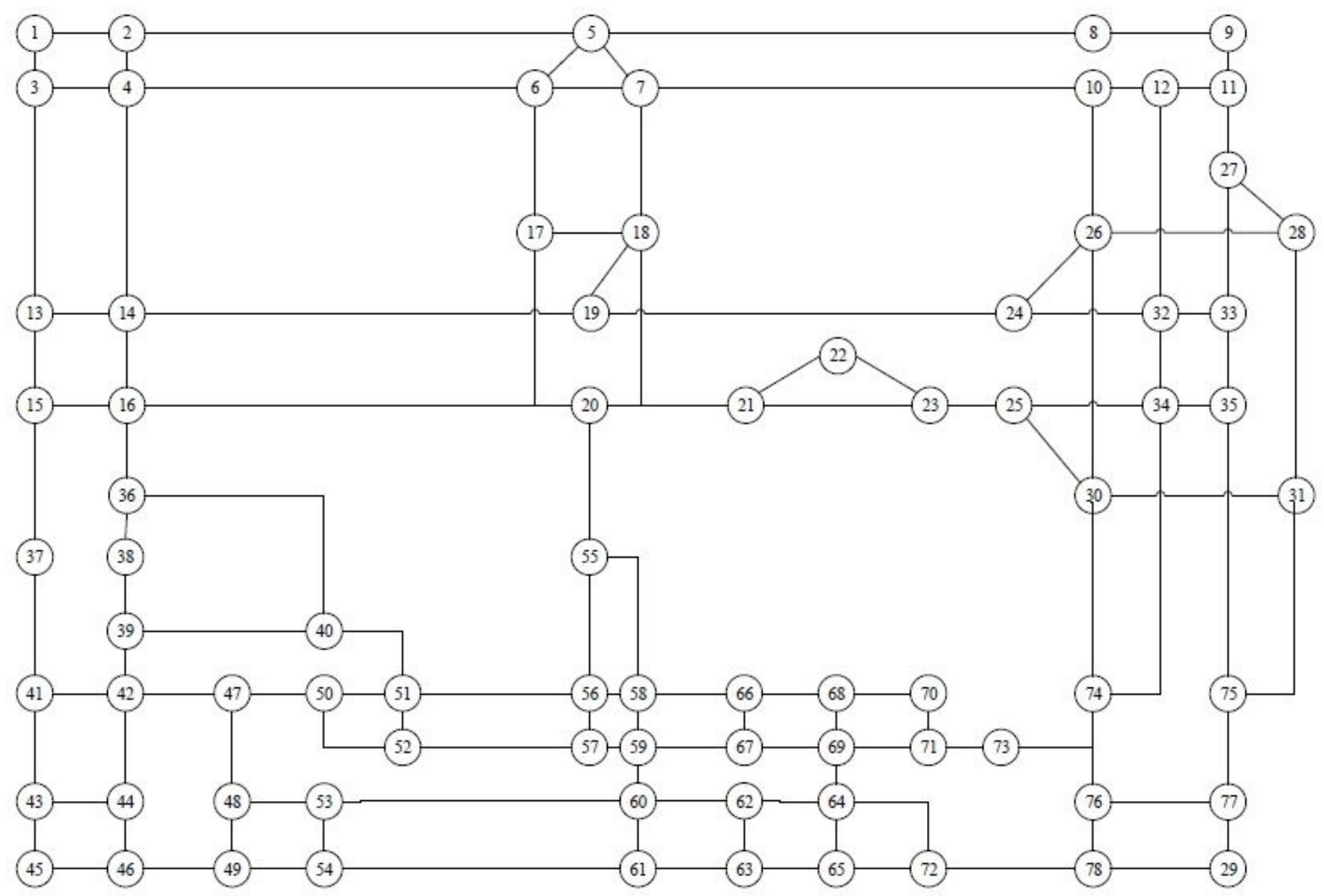

Figure 2

The network topology of the study area 


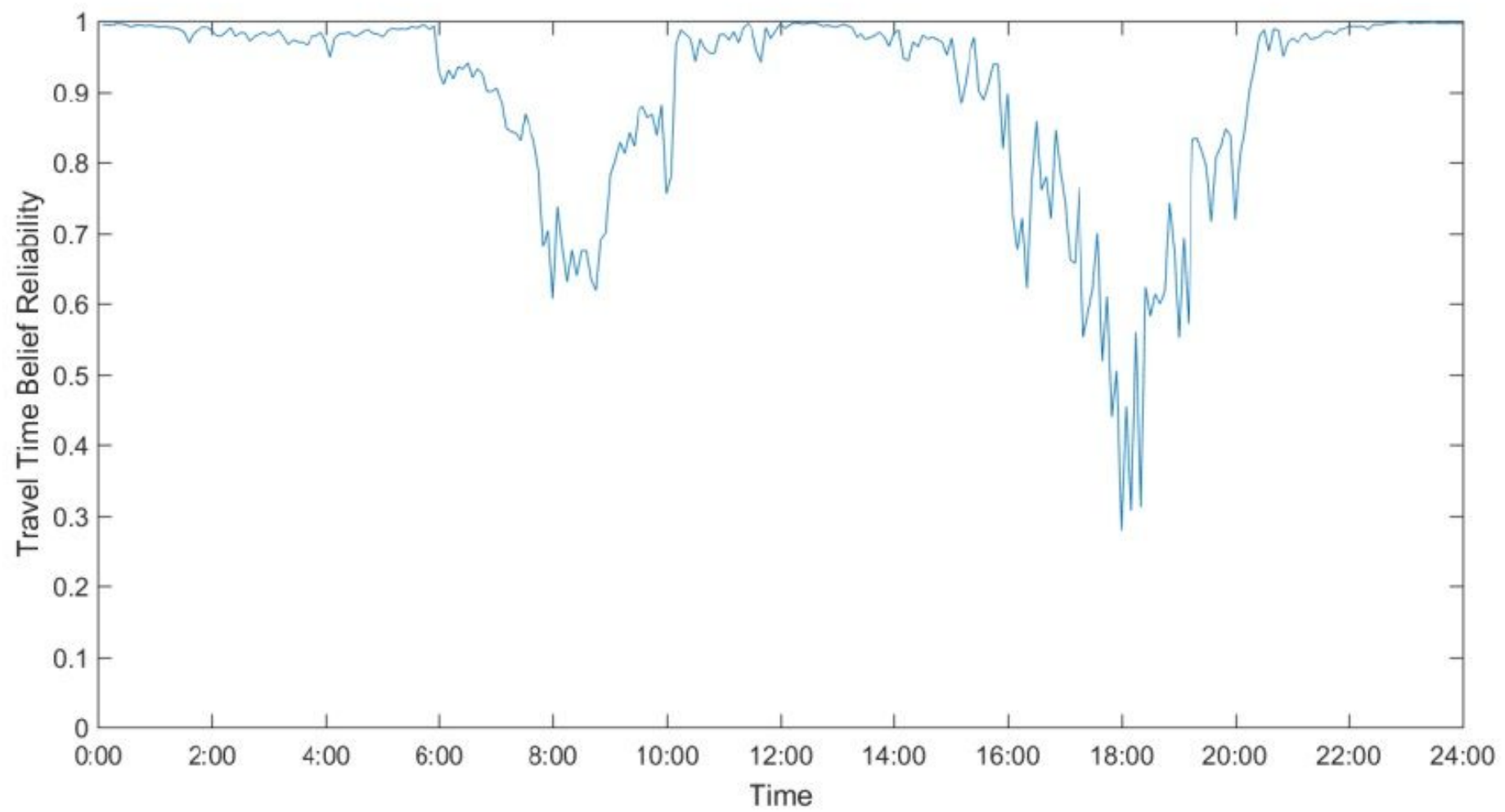

Figure 3

The variation of OD pair travel time belief reliability on working days 